\title{
Function-blocking autoantibodies to the melanin-concentrating hormone receptor in vitiligo patients
}

\author{
Raju VSRK Gottumukkala ${ }^{1}$, Nikos G Gavalas ${ }^{1}$, Samia Akhtar ${ }^{1}$, Russell A Metcalfe ${ }^{1}$, \\ David J Gawkrodger ${ }^{2}$, John W Haycock ${ }^{3}$, Philip F Watson ${ }^{1}$, Anthony P Weetman ${ }^{1}$ \\ and Elizabeth Helen Kemp ${ }^{1}$
}

${ }^{1}$ Division of Clinical Sciences (North), University of Sheffield, Sheffield, UK; ${ }^{2}$ Department of Dermatology, Royal Hallamshire Hospital, Sheffield, UK and ${ }^{3}$ Department of Engineering Materials, University of Sheffield, Sheffield, UK

\begin{abstract}
Vitiligo is a common depigmenting skin disorder resulting from the loss of melanocytes in the cutaneous epidermis. Although the cause of the disease remains obscure, autoimmune mechanisms are thought to be involved. Recently, melanin-concentrating hormone receptor (MCHR)-binding autoantibodies have been identified in vitiligo patients. In the present study, we aimed to determine if MCHR autoantibodies could also affect receptor function either by direct activation or by blocking its response to melanin-concentrating hormone. The results indicated that 10/18 (56\%) vitiligo patient IgG samples inhibited the function of MCHR expressed in a Chinese hamster ovary cell line. In contrast, neither control $(n=20)$ nor SLE patient $(n=10) \lg G$ samples blocked receptor function. Compared with healthy controls, MCHR function-blocking autoantibodies were found at a significantly increased frequency in the vitiligo patient group $(P=0.0004)$. No MCHR-activating autoantibodies were detected in any of the vitiligo patient, SLE patient or control IgG samples that were analysed. In addition, vitiligo patient IgGs were tested for MCHR autoantibodies that could mediate antibodydependent cell-mediated cytotoxicity via the receptor. However, this could only be demonstrated in two vitiligo patient sera. Overall, this work has provided additional evidence that MCHR is a B-cell autoantigen in vitiligo and has demonstrated the existence of MCHR function-blocking autoantibodies further to the receptor-binding autoantibodies previously reported.
\end{abstract}

Laboratory Investigation (2006) 86, 781-789. doi:10.1038/labinvest.3700438; published online 8 May 2006

Keywords: autoimmunity; autoantibody; autoantigen; melanin-concentrating hormone receptor; melanocyte; vitiligo

Vitiligo is an acquired hypomelanotic disorder characterised by circumscribed depigmented macules resulting from the loss of functional melanocytes and of melanin from the epidermis. Large population surveys have shown a prevalence ranging from 0.38 to $1.13 \% .^{1}$ Familial cases of vitiligo are common, indicating a hereditary factor: between 6 and $38 \%$ of vitiligo patients have family members with the disease. ${ }^{2}$ However, studies have indicated that vitiligo is not transmitted by a simple Mendelian mechanism and its inheritance pattern is more consistent with a polygenic trait. ${ }^{3}$

Correspondence: Dr EH Kemp, PhD, Division of Clinical Sciences (North), University of Sheffield, Northern General Hospital, Herries Road, Sheffield S5 7AU, UK.

E-mail: e.h.kemp@sheffield.ac.uk

Received 20 February 2006; revised 13 April 2006; accepted 21 April 2006; published online 8 May 2006
Three major hypotheses for the pathogenesis of vitiligo have been proposed. The neural hypothesis suggests that the accumulation of a neurochemical substance such as a toxin released from nerve endings decreases melanin production as a result of damage to melanocytes. ${ }^{4}$ The biochemical hypothesis implicates the accumulation of toxic intermediate products of melanin synthesis, the breakdown of free radical defence and the build-up of excessive quantities of hydrogen peroxide in the self-destruction of pigment cells. ${ }^{5-7}$ An autoimmune aetiology has also been proposed to explain the depigmentation characteristic of vitiligo. The frequent association of vitiligo with autoimmune diseases, together with studies demonstrating that vitiligo patients have autoantibodies and autoreactive T lymphocytes against melanocyte antigens add support to the theory. ${ }^{8-10}$ It is possible that these different causal factors can act independently or 
together to yield the same effect, namely the disappearance of melanocytes from the skin and this is proposed in the convergence theory. ${ }^{4}$ For example, autoimmunity might arise as a secondary phenomenon following the autodestruction of melanocytes. This might then amplify the damage to pigment cells.

The exact role of anti-melanocyte autoantibodies in the pathogenesis of vitiligo has not been determined, although there is a correlation between both their presence and level and the extent and activity of the disease. ${ }^{11,12}$ Furthermore, sera from vitiligo patients can induce damage to human melanocytes in vitro, by antibody-dependent cellular cytotoxicity and complement fixation and, in vivo, following passive immunisation of nude mice grafted with human skin. ${ }^{13,14}$ Reported humoral autoantigens in vitiligo include tyrosinase ${ }^{15-17}$ tyrosinase-related protein $1,{ }^{18}$ tyrosinase-related protein $2,{ }^{19}$ Pmel $17,{ }^{20}$ and transcription factor SOX10. ${ }^{21}$ Ultimately, autoantibodies against pigment cells may be a marker for autoreactive cytotoxic $\mathrm{T}$ lymphocytes that specifically recognise melanocyte differentiation antigens and that have been detected in both the peripheral blood and peri-lesional skin of individuals with vitiligo. ${ }^{10,22,23}$

Recently, the melanin-concentrating hormone receptor (MCHR) has been identified as a B-cell autoantigen in patients with vitiligo. ${ }^{24}$ Immunoreactivity to the receptor was demonstrated in $16.4 \%$ of vitiligo patient sera in a radiobinding assay and at least some of the MCHR antibody-binding activity blocked $\mathrm{MCH}$ binding in a competitive manner. ${ }^{24}$ MCHR is a G-protein-coupled receptor and its ligand, melanin-concentrating hormone (MCH), both stimulates increases in intracellular calcium and antagonises the regulatory effects of $\alpha$-melanocyte-stimulating hormone $(\alpha-\mathrm{MSH}){ }^{25}$ In pigment cells, stimulation of the receptor with $\mathrm{MCH}$ can downregulate $\alpha$-MSH-induced melanogenesis. ${ }^{26}$

The aim of the present study was to determine if MCHR autoantibodies affect the activity of the receptor as such autoantibodies are more likely to have a role in disease pathogenesis than autoantibodies that simply bind MCHR without affecting its function. Indeed, in several autoimmune disorders, autoantibodies have been demonstrated to affect the functioning of cell receptors resulting in disease manifestations. For example, thyroid-stimulating autoantibodies activate the thyrotropin receptor and elicit hyperthyroidism in Graves' disease by mimicking the binding of thyroid-stimulating hormone and autoantibodies directed against the acetylcholine receptor block the acetylcholinebinding site and provoke accelerated receptor degradation causing myasthenia gravis.. ${ }^{27,28}$ In addition, MCHR autoantibodies were investigated for antibody-dependent cell-mediated cytotoxicity (ADCC) as sera from vitiligo patients have been shown to induce damage to human melanocytes in vitro by this mechanism. ${ }^{13}$

\section{Materials and methods}

\section{Patient and Controls}

Nine vitiligo patients (five male, four female; mean age: 47 years with range $23-75$ years; mean disease duration: 10 years with range $1-37$ years; mean age at disease onset: 38 years with range $<1-73$ years) with MCHR-binding autoantibodies were used in this study. ${ }^{24}$ Vitiligo was classified as peri-orificial, one; symmetrical/peri-orificial, one and symmetrical, seven. Seven patients had no personal or family history of autoimmune disease and two patients had autoimmune thyroid disease. In addition, nine vitiligo patients (three male, six female; mean age: 52 years with range 27-77 years; mean disease duration: 15 years with range 1-36 years; mean age at disease onset: 37 years with range 10-70 years) with no MCHR-binding autoantibodies were included. ${ }^{24}$ In these patients, vitiligo was classified as peri-orificial, one; symmetrical, eight, and two individuals had active vitiligo. Seven patients had no personal or family history of autoimmune disease and two patients had autoimmune thyroid disease. None of the vitiligo patients had autoantibodies to the thyroid-stimulating hormone (TSH) receptor. In total, 10 patients (one male and nine female subjects; mean age: 45 years with range 21-55 years) with systemic lupus erythematosus (SLE) and 20 healthy individuals (nine male and 11 female subjects; mean age: 32 years with range $24-48$ years) with no history of either vitiligo or autoimmune disorders were also included. Aliquots of sera were stored at $-20^{\circ} \mathrm{C}$ before use. The South Sheffield Research Ethics Committee, Sheffield, UK approved this work and all subjects gave informed consent.

\section{IgG Preparation}

Sera were depleted in complement before $\operatorname{IgG}$ preparation by heating at $56^{\circ} \mathrm{C}$ for $30 \mathrm{~min}$. This treatment had no affect upon the immunoreactivity of patient sera to MCHR. IgG was isolated from sera by affinity column chromatography using protein GSepharose 4 Fast Flow (Amersham Biosciences AB, Uppsala, Sweden) according to the manufacturer's instructions. Eluted IgG fractions were extensively dialysed against phosphate-buffered saline (PBS), pH 7.4 (Sigma, Poole, UK), and concentrated using an Amicon Concentrator (Amicon Inc., Beverly, MA, USA). IgG samples were sterilised with a Millex Filter Unit (Millipore Corp., Bedford, MA, USA) and the final concentration was measured by photometry at $280 \mathrm{~nm}$. All $\mathrm{IgG}$ samples were stored at $-20^{\circ} \mathrm{C}$ until required.

\section{Cell Culture}

Chinese hamster ovary (CHO-K1) cells were obtained from the European Collection of Animal Cell 
Cultures (Salisbury, UK). A stable CHO-K1 cell line, expressing MCHR (CHO-MCHR), was kindly given by Dr Emmanuel Burgeon (Euroscreen, Brussels, Belgium). A CHO-K1 cell line expressing the TSH receptor (JPO9) was available in the laboratory. ${ }^{29}$ Cells were cultured in Ham's F12 medium with 10\% $(\mathrm{v} / \mathrm{v})$ fetal calf serum, $2 \mathrm{mM}$ L-glutamine, $50 \mathrm{U} / \mathrm{ml}$ penicillin $\mathrm{G}$ and $50 \mu \mathrm{g} / \mathrm{ml}$ streptomycin (all from Invitrogen, Paisley, UK) at $37^{\circ} \mathrm{C}$ in a $5 \% \mathrm{CO}_{2}$ incubator. For culturing the stable cell lines, geneticin sulphate (Invitrogen) was included in the growth medium at $400 \mu \mathrm{g} / \mathrm{ml}$.

\section{Measurement of Intracellular Calcium}

The response of MCHR expressed in CHO-K1 cells to $\mathrm{MCH}$ was assessed directly by measuring changes in intracellular calcium levels using fluorimetry, as previously described. ${ }^{30}$ Both CHO-K1 and CHOMCHR cells were plated out on glass coverslips in six-well plates at $5.0 \times 10^{5}$ cells/well in $2.5 \mathrm{ml}$ of culture medium and incubated at $37^{\circ} \mathrm{C}$ for 2 days. Cell monolayers (used at $80 \%$ confluence) were then incubated for $30 \mathrm{~min}$ at $37^{\circ} \mathrm{C}$ with the fluorescent dye FURA-2/AM (Merck Biosciences, Nottingham, UK) at $4 \mu \mathrm{M}$ in balanced salt solution (BSS) containing $135 \mathrm{mM} \mathrm{NaCl}, 4.5 \mathrm{mM} \mathrm{KCl}, 1.5 \mathrm{mM} \mathrm{CaCl}$, $0.5 \mathrm{mM} \mathrm{MgCl} 2,5.6 \mathrm{mM}$ glucose and $10 \mathrm{mM}$ HEPES ( $\mathrm{pH}$ 7.4) and then rinsed twice with BSS. Subsequently, the coverslips of cells were placed in a Kontron SFM 25 Fluorimeter (Kontron Instruments Ltd, Watford, UK) across the diagonal of a 1-cm quartz cuvette being supported on a plastic bridge within the cuvette that allowed a small magnetic stirrer to be used to give a continuous mixing of the cuvette contents. A stable baseline of fluorescence was obtained by adding BSS to the cells. Additions of MCH (Alpha Diagnostics, San Antonio, TX, USA) were then made to different concentrations ranging from $10^{-12}$ to $10^{-7} \mathrm{M}$ along with the adenosine agonist N6-(L-2-phenylisopropyl)-adenosine (PIA) at $10 \mu \mathrm{M}$. Changes in intracellular calcium levels following MCH stimulation were detected by measuring changes in relative fluorescence intensity vs time by exciting cells at $335 \mathrm{~nm}$ and collecting emissions at $490 \mathrm{~nm}$.

\section{Assays for MCHR Autoantibodies}

For detecting autoantibodies that activated MCHR, IgG samples were added to CHO-MCHR cells and changes in intracellular calcium levels measured by fluorimetry as detailed above. Briefly, a stable baseline of fluorescence was obtained by adding BSS to FURA-2/AM-labelled CHO-MCHR cells. Samples of IgG from controls $(n=20)$, vitiligo patients $(n=18)$ and SLE patients $(n=10)$ were then added sequentially to either 300 or $1000 \mu \mathrm{g} / \mathrm{ml}$ along with $10 \mu \mathrm{M}$ PIA and intracellular calcium levels monitored. Each IgG sample was tested at each concentration in duplicate in four separate experiments. Duplicate samples of both CHO-MCHR and CHO-K1 cells were stimulated with $\mathrm{MCH}$ in each experiment as positive and negative controls, respectively.

For detecting autoantibodies that blocked the activity of MCHR, CHO-MCHR cells were preincubated with IgG samples and changes in intracellular calcium levels in response to MCH measured by fluorimetry as detailed above. In brief, IgG samples from controls $(n=20)$, vitiligo patients $(n=18)$ and SLE patients $(n=10)$ were added to FURA-2/AMlabelled CHO-MCHR cells to 10, 50, 100, 150 and $300 \mu \mathrm{g} / \mathrm{ml}$ and incubated for $1 \mathrm{~h}$ at $37^{\circ} \mathrm{C}$ in BSS. The cells were then washed twice in BSS. A stable baseline of fluorescence was then obtained by adding BSS to the cells, which were subsequently stimulated with MCH $\left(10^{-12}-10^{-7} \mathrm{M}\right)$ and PIA $(10 \mu \mathrm{M})$ and changes in intracellular calcium levels were measured. Each IgG sample at each concentration was tested in duplicate in four separate experiments. Duplicate samples of both CHO-MCHR and CHO-K1 cells not incubated with IgG were stimulated with MCH in each experiment as positive and negative controls, respectively.

\section{Cell Membrane Preparation}

CHO-K1, CHO-MCHR and JPO9 cells were grown to confluence in 90-mm culture dishes. Cell monolayers were rinsed with PBS and scraped into icecold lysis buffer containing $10 \mathrm{mM}$ Tris-HCl $(\mathrm{pH}$ 7.4), $1 \mathrm{mM}$ EDTA, $1 \mathrm{mM}$ EGTA, $0.25 \mathrm{M}$ sucrose, $1 \mathrm{mM}$ dithiothreitol and Protease Inhibitor Cocktail (Sigma). The resuspended cells were passed 10 times through a 22-gauge needle and the lysate centrifuged at $10000 \mathrm{~g}$ for $15 \mathrm{~min}$ at $4^{\circ} \mathrm{C}$ to remove cellular debris. The supernatants were centrifuged at $45000 \mathrm{~g}$ for $1 \mathrm{~h}$ at $4^{\circ} \mathrm{C}$ and the membrane pellets resuspended in $5 \mathrm{mM}$ HEPES $(\mathrm{pH} 7.4)$ containing Protease Cocktail Inhibitor. Protein concentrations were determined by the Bio-Rad Protein Assay Kit (Biorad Laboratories Ltd, Hemel Hempstead, UK) and supernatant volumes were adjusted to equalise protein concentrations. Membrane aliquots were stored at $-80^{\circ} \mathrm{C}$ until required.

\section{Absorption Experiments}

Membranes (20 $\mu \mathrm{g}$ of protein) from CHO-K1, CHOMCHR or JPO9 cells were incubated with $1 \mathrm{mg} / \mathrm{ml}$ vitiligo patient IgG $(n=10)$ at $4^{\circ} \mathrm{C}$ for $2 \mathrm{~h}$ in buffer containing $150 \mathrm{mM} \mathrm{NaCl}, 10 \mathrm{mM}$ Tris-HCl $(\mathrm{pH}$ 7.4), $1 \%(\mathrm{v} / \mathrm{v}$ ) Triton X-100 and Protease Cocktail Inhibitor. As controls, samples of each patient IgG were also incubated without membranes under the same conditions. Following incubation, the IgG samples were centrifuged at $45000 \mathrm{~g}$ for $1 \mathrm{~h}$ at $4^{\circ} \mathrm{C}$, the supernatants recovered and the IgG concentrations estimated. Each absorbed IgG sample was then 
analysed in duplicate in two separate experiments at $300 \mu \mathrm{g} / \mathrm{ml}$ for MCHR function-blocking activity in CHO-MCHR cells, as described above.

\section{Purification of Peripheral Blood Mononuclear Cells}

Blood $(10 \mathrm{ml})$ from a normal human donor was collected over heparin. The blood was layered over $6 \mathrm{ml}$ of Lymphoprep (Nycomed Pharma AS, Oslo, Norway) and centrifuged at $2800 \mathrm{~g}$ for $20 \mathrm{~min}$. Peripheral blood mononuclear cells (PBMCs) present at the interface were collected and washed twice with PBS by centrifugation at $2000 \mathrm{~g}$ for $5 \mathrm{~min}$. The pellet of PBMCs was resuspended in $2 \mathrm{ml}$ of CHO-K1 cell culture medium and the cells counted in a haemocytometer before making any final adjustments to obtain the required concentration of cells.

\section{Measurement of Antibody-Dependent Cell-Mediated Cytotoxicity}

Confluent monolayers of CHO-MCHR cells were grown in T75 tissue culture flasks. Subsequently, the cells were washed once with PBS and detached from the tissue culture flask after incubation for 5 min at $37^{\circ} \mathrm{C}$ with Cell Dissociation Buffer (Invitrogen). Following centrifugation at $1000 \mathrm{~g}$ for $5 \mathrm{~min}$, cells were resuspended in PBS at a concentration of $1 \times 10^{6} \mathrm{cells} / \mathrm{ml}$ and then labelled with $7.4 \mathrm{MBq}$ of ${ }^{51} \mathrm{Cr}$ (Amersham Pharmacia Biotech, Little Chalfont, UK) by incubation at $37^{\circ} \mathrm{C}$ for $2 \mathrm{~h} .{ }^{51} \mathrm{Cr}$-labelled cells were washed twice with PBS and diluted in culture medium to $1 \times 10^{5}$ cells $/ \mathrm{ml}$. Cells (50- $\mu \mathrm{l}$ aliquots) were then added to each well of a round-bottomed 96-well microtitre plate (Nalge Nunc International, Rochester, NY, USA). To the wells, $100 \mu \mathrm{l}$ of either culture medium, control sera $(n=20)$, vitiligo patient sera $(n=18)$ or SLE patient sera $(n=10)$ were added in triplicate and the plate was incubated for $1 \mathrm{~h}$ at $37^{\circ} \mathrm{C}$. Before use, sera were heat-inactivated by incubation at $56^{\circ} \mathrm{C}$ for $30 \mathrm{~min}$ and diluted at 1:10 in culture medium.

The plate was then centrifuged at $1000 \mathrm{~g}$ for $5 \mathrm{~min}$ and the supernatant removed from each well. Triplicate wells of cells initially incubated with cell culture medium alone were incubated with $150 \mu \mathrm{l}$ of $100 \%$ Triton X-100 in order to determine maximum ${ }^{51} \mathrm{Cr}$ release. To estimate spontaneous ${ }^{51} \mathrm{Cr}$ release, $150 \mu \mathrm{l}$ of cell culture medium were added again in triplicate to cells previously incubated with culture medium alone. To the remaining wells, a $150-\mu \mathrm{l}$ aliquot of PBMC suspension containing $4 \times 10^{5}$ cells/ml was added with the same normal donor being used in all experiments to ensure consistency. The plate was incubated for $4 \mathrm{~h}$ at $37^{\circ} \mathrm{C}$ after which it was centrifuged at $1000 \mathrm{~g}$ for $5 \mathrm{~min}$. The supernatants were removed and the release of ${ }^{51} \mathrm{Cr}$ was counted in an LKB 1470 Rackgamma Liquid Scintillation Analyser (Wallac UK, Milton Keynes, UK).
The percentage lysis by each serum sample was expressed as (sample ${ }^{51} \mathrm{Cr}$-release value-spontaneous ${ }^{51} \mathrm{Cr}$-release value/maximum ${ }^{51} \mathrm{Cr}$-release value-spontaneous ${ }^{51} \mathrm{Cr}$-release value) $\times 100$. Each serum sample was tested in triplicate in two separate experiments and the mean percentage lysis value calculated from these. Percentage lysis values above the set arbitary level of $20 \%$ were considered as a positive indication of ADCC in this assay. ${ }^{31}$

\section{Statistical Analysis}

The frequency of MCHR function-blocking autoantibodies was compared between vitiligo patients and controls using $2 \times 2$ contingency tables and $\chi^{2}$ tests. Yates' correction was applied and $P$-values $<0.05$ (two-tailed) were regarded as significant. Differences in the percentage lysis as a measure of ADCC activity in control and vitiligo patient samples were analysed using the Student's $t$-test and $P$-values $<0.05$ (two-tailed) were regarded as significant.

\section{Results}

\section{Vitiligo Patient IgGs can Block the Function of MCHR}

As binding of MCH to MCHR triggers an increase in intracellular calcium, ${ }^{25}$ the response to $\mathrm{MCH}$ of MCHR expressed in CHO-K1 cells was assessed directly by measuring changes in intracellular calcium levels using fluorimetry. ${ }^{29,30}$ To test for their ability to block the function of MCHR, IgG samples from 18 vitiligo patients, 10 SLE patients and 20 healthy controls were incubated with CHO-MCHR cells before analysing changes in intracellular calcium levels in response to MCH. Each IgG sample was tested in duplicate at 10, 50, 100, 150 and $300 \mu \mathrm{g} / \mathrm{ml}$ in four separate experiments. Duplicate samples of both CHO-MCHR and CHO-K1 cells not incubated with IgG were stimulated with $\mathrm{MCH}$ in each experiment as positive and negative controls, respectively.

A response to $\mathrm{MCH}$ stimulation was not detected in CHO-K1 cells (Figure 1a) but was reproducibly found in CHO-MCHR cells (Figure 1b). Increases in intracellular calcium levels were typically observed within the first $10 \mathrm{~s}$ of $\mathrm{MCH}$ and PIA addition to the cells and responses returned to the original baseline level approximately 30-60 s thereafter. The adenosine agonist PIA,${ }^{30}$ which inhibits the intracellular elevation of adenosine cyclic monophosphate (cAMP), did not itself increase intracellular calcium levels. However, a clearer and more sustained increase in calcium levels was observed in CHOMCR cells when MCH was used in combination with PIA than when MCH was applied alone.

Of the 20 control IgG samples analysed, none blocked the release of intracellular calcium in response to $\mathrm{MCH}$ (Figure 1c; Table 1). Of the 18 vitiligo patient IgG samples tested, eight had no 
effect upon the release of intracellular calcium in response to MCH (Figure 1d; Table 1). In contrast, $10 / 18$ (56\%) vitiligo patient IgG samples blocked the release of intracellular calcium in response to $\mathrm{MCH}$ (Figure 1e; Table 1). For one, six and three IgG samples, respectively, the function of MCHR was blocked at concentrations of 100, 150 and $300 \mu \mathrm{g} / \mathrm{ml}$. Of the 10 SLE patient IgG samples that were tested for MCHR function-blocking reactivity, none was able to block the release of intracellular calcium in response to MCH (Figure 1f; Table 1). Although the
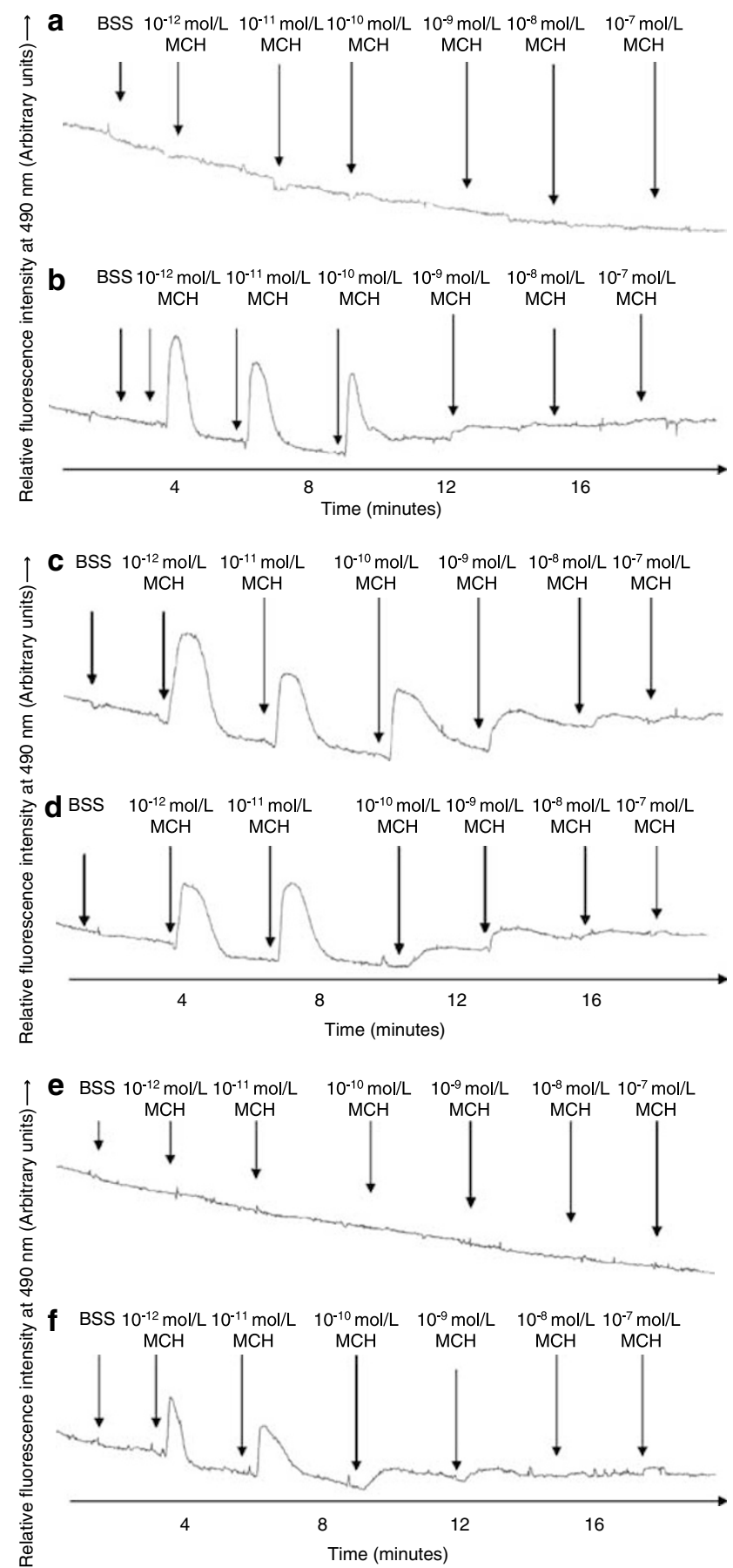

assay was not quantitative, the results were reproducible between experiments. The release of intracellular calcium was only blocked if the MCHRexpressing cells were preincubated for at least $5 \mathrm{~min}$ with vitiligo patient IgG before stimulation with $\mathrm{MCH}$. Concurrent addition of IgG samples with MCH was not sufficient to block receptor function (data not shown).

Overall, MCHR function-blocking IgGs were found at a significantly increased frequency in vitiligo patients compared with healthy controls $(P=0.0004$; Table 1). Among the nine vitiligo patients with MCHR-binding autoantibodies, seven (78\%) had MCHR autoantibodies that blocked receptor function (Table 1). Of nine patients with no MCHR-binding autoantibodies, three (33\%) were demonstrated to have MCHR function-blocking autoantibodies (Table 1). Comparison of healthy controls with vitiligo patients with MCHR-binding autoantibodies indicated that there was a significantly increased frequency of MCHR functionblocking autoantibodies in this patient group

Table 1 Effect of IgG samples on the functioning of MCHR

\begin{tabular}{lccc}
\hline IgG samples & $\begin{array}{c}\text { Number of } \\
\text { samples tested }\end{array}$ & $\begin{array}{c}\text { Number of samples } \\
\text { blocking MCHR } \\
\text { function (\%) }\end{array}$ & P-value $^{\mathrm{a}}$ \\
\hline Total vitiligo & 18 & $10(56 \%)$ & 0.0004 \\
Vitiligo $^{\mathrm{b}}$ & 9 & $7(78 \%)$ & $<0.0001$ \\
Vitiligo $^{\mathrm{c}}$ & 9 & $3(33 \%)$ & 0.0387 \\
Controls $_{\text {SLE }}$ & 20 & 0 & - \\
SLE & 10 & 0 & -
\end{tabular}

${ }^{\mathrm{a}}$ The frequency of MCHR function-blocking autoantibodies was compared between patient and control groups using $2 \times 2$ contingency tables and $\chi^{2}$ tests. Yates' correction was applied and $P$-values $<0.05$ (two-tailed) were regarded as significant.

${ }^{\mathrm{b}} \mathrm{IgG}$ samples from vitiligo patients with MCHR-binding autoantibodies.

${ }^{\mathrm{C}}$ IgG samples from vitiligo patients with no MCHR-binding autoantibodies.

Figure 1 The effect of patient and control IgG on the response of MCHR to MCH. (a) CHO-K1 cells did not respond to stimulation by $\mathrm{MCH}$ as there was no increase in intracellular calcium levels. (b) CHO-MCHR cells responded to stimulation by MCH as there was an increase in intracellular calcium levels. Changes in intracellular calcium levels were also measured in response to MCH in CHO-MCHR cells incubated with either vitiligo patient $(n=18)$, SLE patient $(n=10)$ or control $(n=20)$ IgG samples at 10 , $50,100,150$ and $300 \mu \mathrm{g} / \mathrm{ml}$. All samples were tested in duplicate in four separate experiments. The results of one duplicate in one experiment are shown for IgG samples at $150 \mu \mathrm{g} / \mathrm{ml}$ from one control, two vitiligo patients and one SLE patient. A release of intracellular calcium in response to $\mathrm{MCH}$ was detected in CHOMCHR cells incubated with (c) the control IgG sample, (d) one vitiligo patient IgG sample and (f) the SLE patient IgG sample, indicating that the IgG samples did not block MCH stimulation of the receptor. Following incubation of CHO-MCHR cells with the second vitiligo patient IgG sample (e), no release of intracellular calcium in response to $\mathrm{MCH}$ was demonstrated, indicating that the IgG sample blocked stimulation of the receptor by MCH. 
$(P<0.0001$; Table 1). Similarly, when comparing healthy controls and vitiligo patients with no MCHR-binding autoantibodies, a significant increase in the frequency of function-blocking autoantibodies was noted in the patient cohort $(P=0.0387$; Table 1). In contrast, there was no apparent difference in the frequency of MCHR function-blocking autoantibodies in SLE patients compared with controls (Table 1). No correlation was noted between the detection of MCHR functionblocking autoantibodies and either the clinical subtype of vitiligo, disease activity, age at onset of the disorder, disease duration or the presence of autoimmune disease.

In order to confirm that the function-blocking action of the vitiligo patient IgG samples was antibody-mediated, absorption experiments were undertaken. Samples of IgG from 10 vitiligo patients with MCHR function-blocking autoantibodies were preabsorbed with membrane preparations either from CHO-K1, CHO-MCHR or JPO9 cells. Unabsorbed patient IgGs were also included in each experiment as controls. The effect of preabsorbing patient IgGs on the functioning of MCHR in CHOMCHR cells was then examined in intracellular calcium assays with each IgG tested in duplicate at $300 \mu \mathrm{g} / \mathrm{ml}$. Each preabsorbed IgG sample was analysed in duplicate in two separate experiments to ensure consistency of the results.

In the case of unabsorbed IgGs, all 10 samples still blocked the response of MCHR to $\mathrm{MCH}$ in CHO-MCHR cells as demonstrated in intracellular calcium assays (Figure 2a). Preabsorption of the vitiligo patient IgGs with CHO-K1 or JPO9 membranes did not remove the MCHR function-blocking effects of the IgG samples (Figure 2b and d). In contrast, all 10 IgGs preincubated with CHO-MCHR no longer had an effect upon the release of intracellular calcium in response to MCH (Figure 2c). This indicated that the function of MCHR was unaffected and suggested that MCHR function-blocking autoantibodies had been removed by preabsorption with CHO-MCHR membranes in all 10 of the IgG samples.

\section{Vitiligo Patient IgGs do not Activate MCHR}

To test for MCHR autoantibodies that activated the receptor, IgG samples from 18 vitiligo patients, 10 SLE patients and 20 controls were added to CHOMCHR cells and any effects on intracellular calcium levels were measured by fluorimetry. Each IgG sample was analysed in duplicate at 300 and $1000 \mu \mathrm{g} / \mathrm{ml}$ in four separate experiments. Duplicate samples of CHO-MCHR and CHO-K1 cells were stimulated with MCH in each experiment as positive and negative controls, respectively. None of the samples appeared to stimulate the receptor as no changes in intracellular calcium levels could be detected on addition of IgG to CHO-MCHR cells (data not shown).
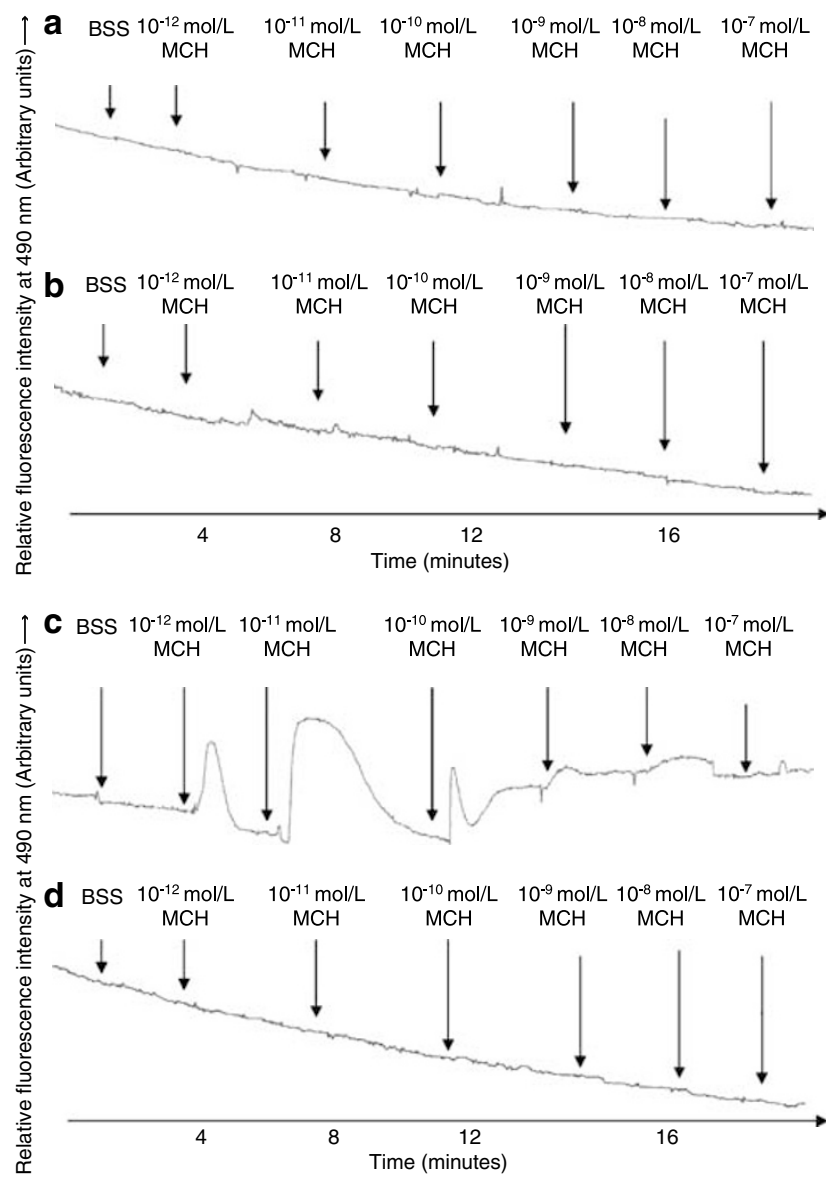

Figure 2 The effect of preabsorption of vitiligo patient IgG on the response of MCHR to MCH. Changes in intracellular calcium levels were measured in response to MCH in CHO-MCHR cells incubated with vitiligo patient $(n=10)$ IgG samples at $300 \mu \mathrm{g} / \mathrm{ml}$. The IgG samples had been preabsorbed with membranes from CHO-K1, CHO-MCHR or JPO9 cells or were untreated. All samples were tested in duplicate in two separate experiments. The results of one duplicate for one vitiligo patient IgG in one experiment are shown for untreated IgG, IgG preabsorbed with CHO-K1 cell membranes, IgG preabsorbed with CHO-MCHR cell membranes and IgG preabsorbed with JPO9 cell membranes. There was no release of intracellular calcium in response to MCH in CHO-MCHR cells incubated with (a) untreated vitiligo IgG, (b) vitiligo patient IgG preabsorbed with CHO-K1 cell membranes and (d) vitiligo patient IgG preabsorbed with JPO9 cell membranes, indicating that the IgG sample blocked the stimulation of the receptor by MCH. Following incubation of CHO-MCHR cells with vitiligo patient IgG preabsorbed with CHO-MCHR cell membranes (c), there was an increase in intracellular calcium demonstrating that the IgG sample did not block the stimulation of the receptor by $\mathrm{MCH}$.

\section{Vitiligo Patient IgGs do not Mediate ADCC Via MCHR}

Autoantibodies to MCHR were investigated for ADCC in ${ }^{51} \mathrm{Cr}$ release assays with CHO-MCHR cells. ${ }^{31}$ Control $(n=20)$, vitiligo patient $(n=18)$ and SLE patient $(n=10)$ sera were incubated with ${ }^{51} \mathrm{Cr}$-labelled CHO-MCHR cells. After washing to remove any unbound antibody, the labelled cells were incubated with PBMCs. These act as effector cells causing lysis and ${ }^{51} \mathrm{Cr}$ release on interaction with any MCHR antibody that might be bound to the 
labelled cells via MCHR. Spontaneous ${ }^{51} \mathrm{Cr}$ release and maximum ${ }^{51} \mathrm{Cr}$ release were estimated in each experiment. Sera were tested in triplicate in two separate experiments.

The results indicated that the percentage lysis resulting from incubation of labelled target cells with effector cells alone ranged from 0.21 to $0.35 \%$. The mean percentage lysis initiated by the control serum samples was $7.12 \%$ with a range of $2.04-$ $12.12 \%$. As all percentage lysis values were below the arbitary set level of $20 \%,{ }^{31}$ none of the control sera was considered positive for ADCC activity. Equally, none of the SLE patient sera tested contained positive ADCC activity against ${ }^{51} \mathrm{Cr}-$ labelled CHO-MCHR cells as again all percentage lysis values were below the arbitary set level of $20 \%$. The mean percentage lysis in the SLE patient group was $7.63 \%$ with a range of $3.12-12.97 \%$. No significant difference was evident between the percentage lysis values of the control group and those of the SLE patient group $(P=0.246)$.

The mean percentage lysis in the vitiligo patient group was $8.21 \%$ with a range of $3.79-25.10 \%$. No significant difference was evident between the percentage lysis values of the control group and those of the vitiligo patient cohort $(P=0.457)$. Only two vitiligo patient samples had percentage lysis values above the arbitary set level of $20 \%$ at 25.10 and $23.94 \%$ and were, therefore, considered positive for ADCC activity. Both patients had symmetrical-type vitiligo, no other autoimmune disease and were positive for both MCHR-binding and receptor function-blocking autoantibodies. However, a correlation between patient characteristics and ADCC activity could not be concluded as other vitiligo patients with the same profile did not appear positive for ADCC activity.

\section{Discussion}

Previously, MCHR was identified as an autoantigen in patients with vitiligo. ${ }^{24}$ Autoantibody reactivity against the receptor was demonstrated in $16.4 \%$ of vitiligo patient sera in a radiobinding assay. The aim of the present study was to determine if MCHR autoantibodies could affect the activity of the receptor, as such autoantibodies are more likely to have a role in disease pathogenesis than autoantibodies that simply bind MCHR. Here, we describe vitiligo patient IgGs that block the functioning of MCHR. Overall, 56\% (10/18) of the vitiligo patient IgGs tested inhibited MCHR function as determined by a block in the release of intracellular calcium in response to $\mathrm{MCH}$ in $\mathrm{CHO}-\mathrm{MCHR}$ cells. Comparing healthy controls and vitiligo patients, MCHR function-blocking autoantibodies were found at a significantly increased frequency in the vitiligo patient group $(P=0.0004)$. In SLE patient IgG samples, which were tested because individuals with this disease generally display a wide variety of autoanti- body reactivity, no MCHR function-blocking autoantibodies were detected, indicating that these receptor autoantibodies are not a general feature of patients with autoimmune disease. In addition, absorption experiments indicated that the receptor autoantibodies were specific for MCHR: receptor function-blocking activity was only removed from patient IgG samples by CHO-MCHR cell membranes and not by JPO9 cell membranes. Interestingly, autoantibodies that block the function of cell receptors are a feature of several autoimmune disorders including autoimmune thyroid disease and myasthenia gravis. ${ }^{32,33}$ Furthermore, specific receptor-activating autoantibodies can also cause autoimmune disease including Graves' disease and autoimmune hypoparathyroidism. ${ }^{34,35}$ However, MCHR-activating autoantibodies were not detected in any of the control or vitiligo patient IgG samples.

Among the nine vitiligo patients with MCHRbinding autoantibodies as measured in a radiobinding assay, ${ }^{24}$ seven contained MCHR autoantibodies that blocked receptor function. In the two patients that only contained receptor-binding autoantibodies, it is probable that the MCHR autoantibodies bind at a site on the receptor that has no effect upon its functionality. In addition, of nine patients with no MCHR-binding autoantibodies, ${ }^{24}$ three were demonstrated to have MCHR function-blocking autoantibodies. The existence of antibodies that do not bind to a receptor but appear to inhibit its function is not unprecedented. Similar findings have been documented for TSH receptor antibodies in Graves' hyperthyroidism: as many as one-third of Graves' disease sera that were positive for TSH receptor-blocking antibodies in a TSH-binding inhibition assay failed to bind to in vitro-translated TSH receptor. ${ }^{36}$ Comparable results have also been reported for binding and blocking autoantibodies to the sodium-iodide symporter in autoimmune thyroid disease. ${ }^{37}$ It may be that the in vitro-translated protein used in the MCHR-radiobinding assay ${ }^{24}$ failed to bind some MCHR autoantibodies owing to incorrect folding of the molecule or the absence of a normal pattern of glycosylation. Indeed, absorption experiments in which MCHR function-blocking reactivity could be specifically removed from all 10 patients with CHO-MCHR membranes suggested that not only was the blocking of receptor function antibody-mediated but also that some MCHR autoantibodies do recognise the receptor in a native environment.

There is evidence that the sera of patients with vitiligo can induce cultured human melanocytes to detach from their cell matrix and can cause direct cytotoxic damage to pigment cells by ADCC. ${ }^{13}$ In this study, MCHR autoantibodies in only two vitiligo patient sera appeared to exhibit ADCC reactivity. It is possible that low expression of MCHR on the surface of CHO-MCHR cells and/or low levels of MCHR autoantibodies in the vitiligo patient samples might mean that ADCC reactivity of 
receptor autoantibodies remains undetected. Overall, the frequency of ADCC reactivity in the vitiligo patient group did not differ significantly from the control population.

The present work has provided additional evidence that MCHR is a B-cell autoantigen in vitiligo by detecting MCHR function-blocking autoantibodies in vitiligo patients further to the receptor binding autoantibodies previously reported. ${ }^{24}$ The effect of MCHR autoantibodies on the function of the receptor in melanocytes either in vitro or in vivo has yet to be determined. Stimulation of MCHR in cultured human melanocytes with MCH can downregulate the actions of $\alpha-\mathrm{MSH}$, including the production of melanin, suggesting that the $\mathrm{MCH} /$ MCHR signalling pathway might have a role with the melanocortins in regulating melanocyte function. ${ }^{26}$ If a blocking effect upon the functioning of the receptor, as shown here in an MCHR-expressing cell line, also occurs in pigment cells, it could disrupt normal melanocyte behaviour. Furthermore, it will be necessary to investigate T-cell reactivity to the receptor as many observations provide evidence of T-cell involvement in vitiligo aetiology. ${ }^{10,22,23}$ Overall, the study is supportive of there being an autoimmune component to the aetiology of vitiligo.

\section{Acknowledgements}

We thank Dr Emmanuel Burgeon (Euroscreen, Brussels, Belgium) for providing the stable CHOK1 cell line expressing MCHR. This work was supported by The Sheffield Hospitals' Charitable Trust (Grant 7830 to EHK, APW and DJG), the British Skin Foundation (Grant 501 to EHK, APW and DJG), the Vitiligo Society (Grant to EHK, APW and DJG) and the Sheffield University Research Committee (Grant to EHK).

\section{Duality of interest}

None declared.

\section{References}

1 Howitz J, Brodthagen H, Schwartz M, et al. Prevalence of vitiligo: epidemiological survey on the Isle of Bornholm, Denmark. Arch Dermatol 1977;113:47-52.

2 Ortonne JP, Mosher DB, Fitzpatrick TB. Vitiligo and other hypomelanoses of hair and skin. In: Ortonne JP, Mosher DB, Fitzpatrick TB (eds). Topics in Dermatology. Plenum Medical Book Company: New York, USA, 1983, pp 257-258.

3 Majumder PP, Nordlund JJ, Nath SK. Pattern of familial aggregation of vitiligo. Arch Dermatol 1993;129: 994-998.

4 Le Poole IC, Das PK, van den Wijngaard RMJGJ, et al. Review of the etiopathomechanism of vitiligo: a convergence theory. Exp Dermatol 1993;2:145-153.

5 Pawelek J, Korner A, Bergstrom A. New regulation of melanin biosynthesis and autodestruction of melanoma cells. Nature 1980;286:617-619.
6 Nordlund JJ, Lerner AB. Vitiligo. It is important? Arch Dermatol 1982;118:5-8.

7 Schallreuter KU, Wood JM, Berger J. Low catalase levels in the epidermis of patients with vitiligo. J Invest Dermatol 1991;97:1081-1085.

8 Ochi Y, DeGroot LJ. Vitiligo in Graves' disease. Ann Intern Med 1969;71:935-940.

9 Naughton GK, Eisinger M, Bystryn J-C. Antibodies to normal human melanocytes in vitiligo. J Exp Med 1983;158:246-251.

10 Ogg GS, Dunbar PR, Romero P, et al. High frequency of skin-homing melanocyte-specific cytotoxic T lymphocytes in autoimmune vitiligo. J Exp Med 1998;188: 1203-1208.

11 Naughton GK, Reggiardo MD, Bystryn J-C. Correlation between vitiligo antibodies and extent of depigmentation in vitiligo. J Am Acad Dermatol 1986;15:978-981.

12 Harning R, Cui J, Bystryn J-C. Relation between the incidence and level of pigment cell antibodies and disease activity in vitiligo. J Invest Dermatol 1991;97: 1078-1080.

13 Norris DA, Kissinger RM, Naughton GK, et al. Evidence for immunologic mechanisms in human vitiligo: patients' sera induce damage to human melanocyes in vitro by complement-mediated damage and antibody-dependent cellular cytotoxicity. J Invest Dermatol 1988;90:783-789.

14 Gilhar A, Zelickson B, Ulman Y, et al. In vivo destruction of melanocytes by the IgG fraction of serum from patients with vitiligo. J Invest Dermatol 1995;105:683-686.

15 Song YH, Connor E, Li Y, et al. The role of tyrosinase in autoimmune vitiligo. Lancet 1994;344:1049-1052.

16 Baharav E, Merimski O, Shoenfeld Y, et al. Tyrosinase as an autoantigen in patients with vitiligo. Clin Exp Immunol 1996;105:84-88.

17 Kemp EH, Gawkrodger DJ, MacNeil S, et al. Detection of tyrosinase autoantibodies in vitiligo patients using ${ }^{35}$ S-labelled recombinant human tyrosinase in a radioimmunoassay. J Invest Dermatol 1997;109:69-73.

$18 \mathrm{Kemp}$ EH, Waterman EA, Gawkrodger DJ, et al. Autoantibodies to tyrosinase-related protein-1 detected in the sera of vitiligo patients using a quantitative radiobinding assay. $\mathrm{Br} \mathrm{J}$ Dermatol 1998;139: 798-805.

19 Okamoto $\mathrm{T}$, Irie RF, Fujii $\mathrm{S}$, et al. Anti-tyrosinaserelated protein-2 immune response in vitiligo and melanoma patients receiving active-specific immunotherapy. J Invest Dermatol 1998;111:1034-1039.

$20 \mathrm{Kemp} \mathrm{EH}$, Gawkrodger DJ, Watson PF, et al. Autoantibodies to human melanocyte-specific protein Pmel17 in the sera of vitiligo patients: a sensitive and quantitative radioimmunoassay (RIA). Clin Exp Immunol 1998;114:333-338.

21 Hedstrand H, Ekwall O, Olsson MJ, et al. The transcription factors SOX9 and SOX10 are vitiligo autoantigens in autoimmune polyendocrine syndrome type I. J Biol Chem 2001;276:35390-35395.

22 Lang KS, Caroli CC, Muhm A, et al. HLA-A2 restricted, melanocyte-specific CD8+ T lymphocytes detected in vitiligo patients are related to disease activity and are predominantly directed against MelanA/MART1. J Invest Dermatol 2001;116:891-897.

23 Palermo B, Campanelli R, Garbelli S, et al. Specific cytotoxic $\mathrm{T}$ lymphocyte responses against MelanA/ MART1, tyrosinase and Gp100 in vitiligo by the use of major histocompatibility complex/peptide tetramers: 
the role of cellular immunity in the etiopathogenesis of vitiligo. J Invest Dermatol 2001;117:326-332.

$24 \mathrm{Kemp} \mathrm{EH}$, Waterman EA, Hawes BE, et al. The melanin-concentrating hormone receptor 1, a novel target of autoantibody responses in vitiligo. J Clin Invest 2002;109:923-930.

25 Hawes BE, Kil E, Green B, et al. The melaninconcentrating hormone receptor couples to multiple $\mathrm{G}$ proteins to activate diverse intracellular signaling pathways. Endocrinology 2000;141:4524-4532.

26 Hoogduijn MJ, Ancans J, Suzuki I, et al. Melaninconcentrating hormone and its receptor are expressed and functional in human skin. Biochem Biophys Res Commun 2002;296:698-701.

27 Weetman AP, McGregor AM. Autoimmune thyroid disease: further developments in our understanding. Endocr Rev 1994;15:788-830.

28 Hoedemaekers AC, van Breda Vriesman PJ, De Baets $\mathrm{MH}$. Myasthenia gravis as a prototype autoimmune receptor disease. Immunol Res 1997;16:341-354.

29 MacNeil S, Munroe DS, Metcalfe R, et al. An investigation of the ability of TSH and Graves' immunoglobulin $\mathrm{G}$ to increase intracellular calcium in human thyroid cells, rat FRTL-5 thyroid cells and eukaryotic cells transfected with the human TSH receptor. J Endocrinol 1994;143:527-540.

30 Metcalfe RA, Findlay C, Robertson WR, et al. Differential effect of thyroid-stimulating hormone (TSH) on intracellular free calcium and cAMP in cells transfected with the human TSH receptor. J Endocrinol 1998;157:415-424.
31 Metcalfe RA, Oh YS, Stroud C, et al. Analysis of antibody-dependent cell-mediated cytotoxicity in autoimmune thyroid disease. Autoimmunity 1997;25: 65-72.

32 Arikawa K, Ichikawa Y, Yoshida T, et al. Blocking type antithyrotropin receptor antibody in patients with nongoitrous hypothyroidism: its incidence and characteristics of action. J Clin Endocrinol Metab 1985;60:953-959.

33 Hara $\mathrm{H}$, Hayashi $\mathrm{K}$, Ohta $\mathrm{K}$, et al. Detection and characterization of blocking-type anti-acetylcholine receptor antibodies in sera from patients with myasthenia gravis. Clin Chem 1993;39:2053-2057.

34 Schott M, Scherbaum WA, Morgenthaler NG. Thyrotropin receptor autoantibodies in Graves' disease. Trends Endocrinol Metab 2005;16:243-248.

35 Kifor O, McElduff A, Leboff MS, et al. Activating antibodies to the calcium-sensing receptor in two patients with autoimmune hypoparathyroidism. J Clin Endocrinol Metab 2004;89:548-556.

36 Morgenthaler NG, Tremble J, Huang G, et al. Binding of antithyrotropin receptor autoantibodies in Graves' disease serum to nascent, in vitro translated thyrotropin receptor: ability to map epitopes recognised by antibodies. J Clin Endocrinol Metab 1996;81: 700-706.

37 Ajjan RA, Kemp EH, Waterman EA, et al. Detection of binding and blocking autoantibodies to the human sodium-iodide symporter in patients with autoimmune thyroid disease. J Clin Endocrinol Metab 2000;85:2020-2027. 